\title{
Capecitabine-Induced Coronary Vasospasm
}

\author{
Danish Henry Francine Rudzik Allison Butts Aju Mathew \\ Markey Cancer Center, University of Kentucky, Lexington, Ky., USA
}

\section{Keywords}

Capecitabine $\cdot 5$-Fluorouracil $\cdot$ Chest pain $\cdot$ Coronary artery disease $\cdot$ Breast cancer

\begin{abstract}
Capecitabine, an oral prodrug of 5-fluorouracil (5-FU), is approved for early-stage and advanced colorectal cancer and metastatic breast cancer. Cardiotoxicity of 5-FU is well described in the literature. However, cardiac adverse effects of capecitabine are poorly described. We report a case of coronary vasospasm induced by capecitabine. A 41-year-old female with metastatic breast cancer presented with chest pain 3 days after starting capecitabine. The chest pain was relieved by rest and exacerbated by exertion. Her physical examination was unremarkable except for a rapid heart rate of $100 \mathrm{bpm}$. Electrocardiogram test showed no acute ischemic changes. Troponin tests were negative. CT angiography of the chest was negative for acute pulmonary embolism. An echocardiogram showed a left ventricular ejection fraction of $60 \%$ without any wall motion abnormalities. The chest pain resolved with aspirin and analgesic use. She was discharged following an inconclusive cardiac workup. Further use of capecitabine was discontinued.

(C) 2016 The Author(s) Published by S. Karger AG, Basel
\end{abstract}

\section{Introduction}

Capecitabine (Xeloda) is an oral fluoropyrimidine antimetabolite, which is converted by thymidine phosphorylase to 5 -fluorouracil (5-FU). This enzyme is preferentially expressed 


\section{Case Reports in Oncology}

Henry et al.: Capecitabine-Induced Coronary Vasospasm

by tumor cells rendering capecitabine highly selective for sensitive tumor cells [1]. It is enlisted in the WHO List of Essential Medicines and is currently approved for early-stage and advanced colorectal cancer and metastatic breast cancer. Cardiotoxicity with fluoropyrimidine-based treatment is occasionally observed, and could even be fatal [2, 3]. Although 5-FU is known to cause cardiotoxicity, capecitabine-induced cardiac side effects are poorly described in the literature. Herein, we report a case of coronary vasospasm induced by capecitabine in a patient with no pre-existing coronary artery disease (CAD) or risk factors.

\section{Case Presentation}

A 41-year-old female with metastatic breast cancer presented to the emergency department with chest pain of 1 day duration. The chest pain started gradually, and was relieved by rest and exacerbated by exertion. She was started on capecitabine therapy 3 days before the onset of chest pain. She denied shortness of breath, nausea, vomiting, diaphoresis, fever, syncope, heartburn, and peripheral edema. The patient did not have a history of CAD or had known risk factors for CAD. She denied the use of cocaine. The patient did not describe symptoms suggestive of panic attack. On examination, her heart rate was $100 \mathrm{bpm}$ and regular. Her physical examination was otherwise unremarkable.

Five years before the current event, she underwent mastectomy and axillary lymph node dissection for a stage III (pT2N3) breast cancer. The tumor was ER+, PR+, HER2+, and she received adjuvant chemotherapy and trastuzumab, followed by tamoxifen. She was diagnosed with metastatic disease in the liver in 2014, and was started on trastuzumab, pertuzumab, and docetaxel. Fifteen months later, she had disease progression in the liver and was switched to trastuzumab-emtansine. Nine months later, she progressed again, and therapy was changed to lapatinib and capecitabine $(1,500 \mathrm{mg}$ b.i.d.). Three days after starting capecitabine, she experienced acute-onset chest pain.

Electrocardiogram (ECG) testing showed normal sinus rhythm and no changes suggestive of coronary ischemia. Cardiac enzymes were negative. Complete blood count and chemistry panel were within normal range. CT angiography of the chest did not reveal any evidence of pulmonary embolism. A transthoracic echocardiogram documented normal cardiac function and a left ventricular ejection fraction of $60 \%$. Her chest pain resolved after aspirin and analgesics were administered upon admission in the ER. A cardiology opinion was sought for possible angiography, but was not undertaken because of the patient's improving condition. She was discharged following complete resolution of chest pain. Oncology was consulted during the hospitalization, and capecitabine therapy was discontinued. Since then, she has had no complaints of chest pain. Repeat echocardiogram was normal as well.

\section{Discussion}

The selective anti-tumor action of capecitabine increases its tolerability. However, capecitabine and its parental compound may induce cardiotoxicity, depending on the dose, cardiac comorbidity and schedule of chemotherapy [4]. Capecitabine cardiotoxicity is thought to occur through the action of 5-FU on the endothelium resulting in production of endothelin-1 and subsequent coronary vasospasm [5].

Patients present with symptoms similar to variant angina, with chest pain at rest and may or may not have ECG changes of ischemia. Exercise-induced ECG changes have been 
observed with capecitabine use [6]. In fact, Goldsmith et al. [7] reported exercise-induced global myocardial ischemia in a patient with normal coronary arteries and left ventricular function who was on capecitabine for recurrent breast cancer.

With regard to onset of chest pain with capecitabine therapy, Wijesinghe et al. [8] reported an acute coronary syndrome in a patient who had been on capecitabine for only 2 days. Goldsmith et al. [7] reported a patient who experienced symptoms due to coronary vasospasm a few days after starting capecitabine. Our patient presented with chest pain 3 days after taking capecitabine. Depending on the dose, cardiac side effects can occur within $24 \mathrm{~h}$ after taking the drug [9].

As for our patient, the resolution of chest pain, normal ECG and troponins ruled out myocardial infarction and dysrhythmias. The recording of ST segment changes on ECG depends on the presence of symptoms at the time of assessment. Our patient's chest pain subsided before ECG was recorded that could have possibly led to the absence of changes suggestive of coronary vasospasm. The normal echocardiogram excluded direct damage to the myocardium or coronary vessels by capecitabine metabolite. It was thought that a coronary angiography and a stress test are not necessary for her management, and therefore it was not obtained.

The cardiotoxicity of fluoropyrimidines manifests as vasospasm, hypertension, ventricular arrhythmias, cardiogenic shock, and even cardiac arrest [2, 5, 10]. Also, there is a higher prevalence of capecitabine cardiotoxicity in patient with CAD. This is because thymidine phosphorylase activity is reported to be expressed in atherosclerotic plaques [11]. Our patient did not have CAD. Of note, 5-FU-mediated cardiotoxicity can occur in patients with no pre-existing CAD [5-7, 12].

In summary, our patient had capecitabine-induced coronary vasospasm in the absence of pre-existing CAD. Coronary vasospasm is reversible with discontinuation of capecitabine. Early recognition of this complication of treatment will help prevent adverse events. Further use of capecitabine must be discontinued to avoid risk for cardiotoxicity.

\section{Statement of Ethics}

The authors have no ethical conflicts to disclose.

\section{Disclosure Statement}

The authors have no conflicts of interest to disclose.

\section{References}

1 Schellens JH: Capecitabine. Oncologist 2007;12:152-155.

-2 Polk A, Vistisen K, Vaage-Nilsen M, Nielsen DL: A systematic review of the pathophysiology of 5fluorouracil-induced cardiotoxicity. BMC Pharmacol Toxicol 2014;15:1.

3 Ang C, Kornbluth M, Thirlwell M, Rajan R: Capecitabine-induced cardiotoxicity: case report and review of the literature. Curr Oncol 2010;17:59-63.

-4 Südhoff T, Enderle M-D, Pahlke M, Petz C, Teschendorf C, Graeven U, et al: 5-Fluorouracil induces arterial vasocontractions. Ann Oncol 2004;15:661-664.

-5 Tsiamis E, Synetos A, Stefanadis C: Capecitabine may induce coronary artery vasospasm. Hellenic J Cardiol 2012;53:320-323. 
6 Camaro C, Danse P, Bosker H: Acute chest pain in a patient treated with capecitabine. Neth Heart J 2009;17:288-291.

-7 Goldsmith YB, Roistacher N, Baum MS: Capecitabine-induced coronary vasospasm. J Clin Oncol 2008;26:3802-3804.

-8 Wijesinghe N, Thompson PI, McAlister H: Acute coronary syndrome induced by capecitabine therapy. Heart Lung Circ 2006;15:337-339.

-9 Schnetzler B, Popova N, Lamb CC, Sappino A: Coronary spasm induced by capecitabine. Ann Oncol 2001;12:723-724.

10 Kosmas C, Kallistratos MS, Kopterides P, Syrios J, Skopelitis H, Mylonakis N, et al: Cardiotoxicity of fluoropyrimidines in different schedules of administration: a prospective study. J Cancer Res Clin Oncol 2008;134:75-82.

11 Boyle JJ, Wilson B, Bicknell R, Harrower S, Weissberg PL, Fan TP: Expression of angiogenic factor thymidine phosphorylase and angiogenesis in human atherosclerosis. J Pathol 2000;192:234-242.

12 Golias C, Dimitriadis G, Dimitriadis D, Graidis C, Dimitrelos I, Tsiakou A, et al: Acute presentation of vasospastic angina induced by oral capecitabine: a case report. J Med Case Rep 2014;8:1. 